\title{
The challenges of using radiological 'tumour response' as an outcome: lessons learned from neo-tango and artemis, two neo-adjuvant chemotherapy breast cancer trials
}

\author{
Louise Hiller $^{1 *}$, Janet Dunn ${ }^{1}$, Anne-Laure Vallier ${ }^{2}$, Clare Blenkinsop¹, Louise Grybowicz², Helen Higgins', \\ Helena Earl $^{3}$
}

From 2nd Clinical Trials Methodology Conference: Methodology Matters

Edinburgh, UK. 18-19 November 2013

\section{Objectives}

To review the measurement methods of radiological 'tumour response' in two clinical trials.

\section{Methods}

Neo-tAnGo and ARTemis are national 800-patient breast cancer trials assessing neo-adjuvant chemotherapy regimens, both with a secondary endpoint of radiological tumour response, assessing treatment effect in terms of change in tumour size. Both trials record radiological sizes at baseline, mid-way through and at the end of the chemotherapy regimens.

Neo-tAnGo (randomising Jan'05 to Sept'07) recorded mammograms and/or ultrasounds and/or MRI scans, identifying up to two breast and one axillary lesion as 'target' lesions. At each of the 3 time-points and for each target lesion, the scan types and largest diameters observed (maximum of two) were reported, along with a clinical judgement of response.

ARTemis (randomising May'09 to Jan'13) recorded ultrasounds only, recording the longest single diameter of each tumour (including axillary tumours) along with total 'tumour bulk' in each breast. At mid- and endchemotherapy, a clinical judgement of response per breast is also recorded.

\section{Results}

In neo-tAnGo, the type of radiological scan used and number of recorded dimensions varied, not only across hospitals but also within-hospitals across patients and across assessment times, creating challenges regarding tumour comparisons. In ARTemis, difficulties existed surrounding the clinical response categorisation and interpretation of 'tumour bulk', and non-adherence to the specified radiological technique raised methodological issues.

\section{Conclusions}

Optimally recording radiological 'tumour response' and making meaningful inter-patient, inter-tumour or intertime-point comparisons is challenging. Specifications regarding the authorised radiological scanning techniques and reporting of findings are imperative in a trial protocol.

\section{Authors' details}

${ }^{1}$ Warwick Clinical Trials Unit, University of Warwick, Coventry, UK. ${ }^{2}$ Cambridge Cancer Trials Centre, Cambridge University Hospitals NHS Foundation Trust, Cambridge, UK. ${ }^{3}$ Department of Oncology, University of Cambridge and NIHR Cambridge Biomedical Research Centre, Cambridge, UK.

Published: 29 November 2013

doi:10.1186/1745-6215-14-S1-P75

Cite this article as: Hiller et al:: The challenges of using radiological 'tumour response' as an outcome: lessons learned from neo-tango and artemis, two neo-adjuvant chemotherapy breast cancer trials. Trials 2013 14(Suppl 1):P75. 